\title{
Reducing Expectations for Antibiotics in Patients With Upper Respiratory Tract Infections: A Primary Care Randomized Controlled Trial
}

Anna Ishani Perera, MBCbB ${ }^{1}$

Mark Greenslade Thomas, $M D^{2,3}$

Keith James Petrie, $P b D^{1}$

Janet Frater, $M B C b B^{4}$

Daniel Dang, $\mathrm{PbD}^{5}$

Kiralee Ruth Schache, MHP'

Amelia Frances Akroyd, MHP'

Stephen Robert Ritchie, $P b D^{2}$

'Department of Psychological Medicine, University of Auckland, Auckland, New Zealand

${ }^{2}$ Department of Molecular Medicine and Pathology, University of Auckland, Auckland, New Zealand

${ }^{3}$ Department of Infectious Diseases, Auckland City Hospital, Auckland, New Zealand

${ }^{4}$ Balmoral Doctors, Auckland, New Zealand

${ }^{5}$ Eastern Institute of Technology, Napier, New Zealand

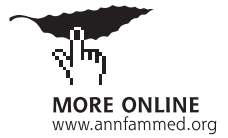

Conflicts of interest: authors report none.

\section{CORRESPONDING AUTHOR}

Anna Ishani Perera

Department of Molecular Medi-

cine and Pathology

Medical and Health Sciences

University of Auckland

85 Park Rd

Grafton, Auckland 1023, New Zealand

anna.perera@auckland.ac.nz

\begin{abstract}
PURPOSE Many family practitioners prescribe antibiotics for patients with upper respiratory tract infections (URTIs) to meet patients' expectations. We evaluated the impact of providing brief tablet-based information about antibiotic treatment of URTIs on patients' expectations for antibiotics and on family practitioners' antibiotic-prescribing behavior.
\end{abstract}

METHODS We performed a 3-arm randomized controlled trial among patients presenting with URTIs at 2 urban family practices in Auckland, New Zealand, during winter 2018. Participants were randomly allocated to view a presentation about the futility of antibiotic treatment of URTIs, the adverse effects associated with antibiotics, or the benefits of healthy diet and exercise (active control), immediately before their consultation. Before and after viewing the presentations, participants used a Likert scale to rate the strength of their belief that antibiotics are effective for treating URTIs and of their desire to be prescribed an antibiotic. Patients reported whether an antibiotic had been prescribed, and pharmacy dispensing records were reviewed to determine whether an antibiotic was dispensed.

RESULTS Participants who viewed either the futility or the adverse effects presentation had greater reductions in their expectations to receive antibiotics than the control group. The mean reduction $(95 \% \mathrm{Cl})$ was $1.1(0.8-1.3)$ for the futility group, 0.7 (0.4-0.9) for the adverse effects group, and 0.1 (0-0.3) for the control group (Cohen $d=0.7 ; P<.001$ ). There was no significant difference among the 3 groups with regard to antibiotic prescribing $(P=.84)$ or dispensing $(P=.43)$.

CONCLUSIONS A brief tablet-based waiting room intervention significantly reduced participants' expectations about receiving antibiotics for URTI immediately before their family practitioner consultation. The intervention did not influence family practitioner prescribing behavior, however.

Ann Fam Med 2021;19:232-239. https://doi.org/10.1370/afm.2672.

\section{INTRODUCTION}

$\mathrm{O}$ veruse of antibiotics drives the emergence and spread of antimicrobial-resistant organisms, which pose a major threat to public health. ${ }^{1}$ Globally, $85 \%$ to $95 \%$ of human antibiotics consumed are prescribed in outpatient settings such as family practice clinics and urgent care facilities. ${ }^{2}$ The majority of non-guideline adherent, or inappropriate, antibiotic prescribing at these sites is for patients with selflimiting upper respiratory tract infections (URTIs), for which antibiotics confer marginal clinical benefit. ${ }^{3,4}$ When antibiotics provide little or no benefit, the risk of antibiotic-related harms far outweigh the potential benefits. ${ }^{5,6}$ Curtailing inappropriate antibiotic prescribing for patients with URTI is necessary to reduce harm and to slow the development of antimicrobial-resistant organisms.

Numerous psychosocial factors influence prescribing of antibiotics for URTIs. One factor that significantly drives inappropriate antibiotic 
prescribing is patients' expectations. Cross-sectional research indicates that the majority of family practitioners would prescribe antibiotics for a URTI to satisfy patient expectations, ${ }^{7}$ despite knowing that this treatment would be ineffective for the patient's illness. ${ }^{8}$ Physicians were more likely to prescribe antibiotics for a hypothetical patient perceived to have high expectations for antibiotics than for a hypothetical patient with low expectations. ${ }^{9}$ Many patients with URTIs expect to receive antibiotics, ${ }^{10,11}$ and many patients underestimate the harms of antibiotics and wrongly believe that these medications are useful for treating URTIs. ${ }^{10,12}$ Therefore, reducing patients' expectations for antibiotic treatment of URTIs could significantly reduce inappropriate antibiotic prescribing. There is little evidence, however, that educational interventions, delivered at the point of care, can significantly change patients' expectations of being prescribed an antibiotic. ${ }^{13}$

The Necessity-Concern Framework posits that patients' medication beliefs and behaviors are determined by the balance between the perceived necessity of a medication for maintaining health, and concern about the consequences of taking the medication. ${ }^{14}$ Using this framework, we devised 2 tablet-based presentations designed to reduce patients' expectations for antibiotics of URTIs.

We conducted a trial in which patients presenting with symptoms of URTI were randomized to view 1 of 3 presentations focused on the futility of antibiotics for an URTI (designed to reduce "necessity" beliefs) or on the risk of antibiotic-related adverse effects (designed to increase "concern" beliefs) or on the benefits of a healthy lifestyle without information about antibiotics or URTI (active control). We measured the effect of the presentations on patients' beliefs that antibiotics are helpful for URTIs, their expectations of being prescribed an antibiotic, and their actual receipt of an antibiotic prescription.

\section{METHODS}

The study was a randomized controlled trial that compared the effects of viewing 2 brief tablet-based educational presentations, designed to reduce expectations for antibiotics in patients with URTIs, with the effects of viewing a control presentation. Participants were recruited from 2 large urban family practices in Auckland, during the winter (July to September) of 2018. The study was conducted in accordance with the Declaration of Helsinki, and the study protocol was granted approval by the Health and Disability Ethics Committee of New Zealand (Ref 17/STH/144) and was registered with the Australia New Zealand Clinical Trials Registry (Trial ID 12617000744358).

\section{Participants}

Patients whose main reason for presentation was symptoms of URTI - cough, earache, sore throat, or nasal congestion (or some combination thereof)—were invited to participate. Based on our previous findings, ${ }^{15}$ we estimated that a behavioral intervention would have a small to moderate effect (Cohen $d=0.35$ ) on patients' expectations for antibiotics. Combined with a power of .80 and an $\alpha$ level of .05, a total sample of 231 was required to detect this effect. Participant attrition at the postconsultation follow-up was estimated to be about $10 \%$; therefore, we aimed to recruit at least 255 total participants (ie, 85 in each group).

In the family practitioner waiting room, research assistants provided participants brief information that their practice was participating in a University of Auckland study. They were not told that the aim of the research was to measure the impact of the various presentations on patients' expectations to receive antibiotics. Adult patients (aged $\geq 18$ years) presenting with symptoms of URTI, and parents of children aged 0 to 7 years presenting with symptoms of URTI, were eligible to participate. Potential participants were excluded if they were attending an appointment with a nurse (there were no nurse prescribers at either practice) or were unable to read, write, or speak English.

\section{Processes}

\section{Prepresentation Questionnaire}

After providing written informed consent, each participant completed a questionnaire. Data collected included participant age, sex, level of education, and self-reported ethnicity; their symptoms (any of cough, sore throat, nasal discharge, or earache); and whether the visit to the family practitioner was for themselves or for their child. Participants used a 7-point Likert scale to indicate their level of agreement with the following statements/questions: (1) "How bad is the cold/ flu that you/your child has today?" (to measure perceived illness severity); (2) "How worried are you about your/your child's cold/flu?" (to measure concerns about the illness); (3) "I think antibiotics are a helpful treatment for cold/flu" (to measure belief in the efficacy of antibiotics for URTIs); and (4) "I wish to receive antibiotics for my/my child's cold/flu" (to measure expectation for antibiotics). Response options ranged from 1 (strongly disagree) to 7 (strongly agree).

\section{Presentation}

After completing the prepresentation questionnaire, participants were randomized to view 1 of 3 presentations on a tablet device. Participants were allocated 1:1:1 without adjustment for prior allocation within the tablet application. Each presentation contained 6 slides, 
and the entire presentation took approximately 1 minute to complete. One presentation focused on the futility of antibiotics for URTIs and contained information about alternative treatments (eg, nasal spray, throat lozenges). The second presentation focused on the potential adverse effects of antibiotics and also contained information about alternative treatments for URTIs. The third (control) presentation promoted healthy lifestyle choices but did not mention antibiotics or URTIs.

\section{Postpresentation Questionnaire}

Immediately after the presentation, participants again used a 7-point Likert scale to indicate their level of agreement with the 2 previous statements addressing belief in efficacy and expectations: (1) "I think antibiotics are a helpful treatment for cold/flu," and (2) "I wish to receive antibiotics for my/my child's cold/flu." Participants then proceeded to their family practitioner consultation. Although the physicians consulting at the practices were aware of the study, they were not informed by the research team whether individual patients had consented to participate or which presentation participating patients had viewed. beliefs in the efficacy of antibiotics for URTIs for each group were compared using the Kruskal-Wallis test. An estimate of the effect size was determined by calculating the Cohen $d$ from the Kruskal-Wallis H statistic. Factors associated with continuing to expect antibiotics after viewing a presentation were identified using multivariate ordinal logistic regression analysis; Likert scores were included as categorical variables.

We used using binomial logistic regression analyses to identify the factors associated with receiving an antibiotic prescription and with having an antibiotic dispensed during the 7 days after the consultation. The multivariate models included factors significantly associated with antibiotic prescription or dispensing after univariate binomial regression analysis, and included the presentation viewed as a factor of interest.

\section{RESULTS}

In total, 325 participants completed both prepresentation and postpresentation questionnaires (Figure 1). Most (94\%) also completed the postconsultation questionnaire. Roughly a third of participants $(28 \%)$ were consulting the family practitioner because their child

\section{Postconsultation}

Immediately after their visit, participants reported whether they had been prescribed an antibiotic and their level of satisfaction (on a scale of 0 to 5) with the consultation. To collect information on whether patients had a prescription dispensed and whether patients may have been prescribed antibiotics by another clinician, all medications dispensed during the 7 days after the study visit were obtained from the National Pharmaceutical Collection database using each participant's unique National Health Index number.

\section{Analysis}

We performed analyses using SPSS 25 (IBM Corp). The primary outcome was the change in participants' expectations of receiving antibiotics for their URTI after viewing a presentation. Changes in each participant's expectations to receive an antibiotic and in

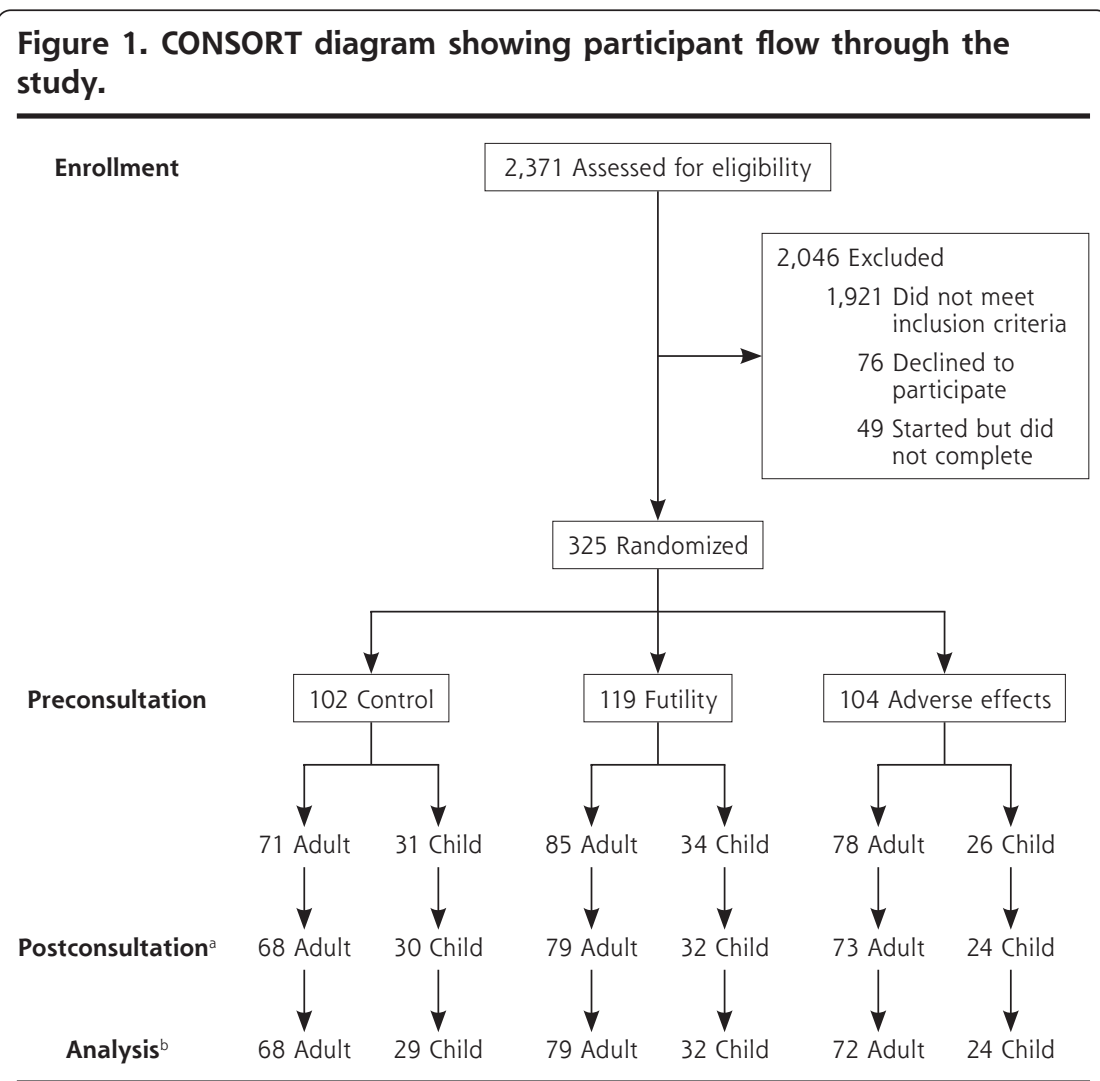

CONSORT $=$ Consolidated Standards of Reporting Trials.

a A total of 19 participants were lost to follow-up at postconsultation ( $N=306$ were followed up).

${ }^{\mathrm{b}}$ An additional 2 participants did not have data available for analysis ( $\mathrm{N}=304$ had complete data for analysis). 
(aged 0 to 7 years) was unwell. There were no significant differences in clinical and demographic characteristics comparing the participants who viewed the futility, adverse effects, and control presentations (Table 1).

\section{Expectations and Beliefs About Antibiotics for URTI}

Likert scores for participants' agreement with the statement "I wish to receive antibiotics for my/my child's cold/flu" before presentation viewing did not differ significantly across the 3 groups viewing different presentations. The mean (SD) score was $3.0(0.3)$ in the futility group, $2.6(0.3)$ in the adverse effects group, and $2.7(0.3)$ in the control group $(P=.20)$.

After viewing the presentation, the mean reduction in score for those who viewed the futility presentation $(1.1 ; 95 \% \mathrm{CI}, 0.8$ to 1.3$)$ or the adverse effect presentation $(0.7 ; 95 \% \mathrm{CI}, 0.4$ to 0.9$)$ was significantly greater than that for those who viewed the control presentation $(0.1 ; 95 \% \mathrm{CI}, 0.0$ to 0.3 ) (Kruskal-Wallis $H=36.7$, Cohen $d=0.7, P<.001$ ) (Figure 2 ). This effect was also observed when analysis was restricted to the parents of the 91 child participants; the mean reduction in score for those who viewed the futility presentation or adverse effect presentation $(0.8 ; 95 \% \mathrm{CI}, 0.5$ to 1.4) was significantly greater than that for those who viewed the control presentation $(0.1 ; 95 \% \mathrm{CI},-0.2$ to 0.3) (Kruskal-Wallis $P<.01$ ).

Similarly, after viewing the presentations, the mean reduction in Likert scores for participants' agreement with the statement "I think antibiotics are a helpful treatment for cold/flu" were significantly greater for those who viewed the futility presentation $(0.9 ; 95 \%$ CI, 0.6 to 1.2$)$ and adverse effect presentation $(0.7$; $95 \% \mathrm{CI}, 0.4$ to 1.1$)$ than for those who viewed the control presentation $(-0.1 ; 95 \% \mathrm{CI},-0.2$ to 0.1$)$ (KruskalWallis $H=31.4$, Cohen $d=0.6, P<.001)$.

\section{Factors Associated With a Continuing Expectation to Receive Antibiotics}

We classified participants who agreed or strongly agreed with the statement "I wish to receive antibiotics for my/my child's cold/ flu" on the postpresentation questionnaire as having a continued expectation for antibiotics. The univariate odds ratios for factors associated with a continued expectation to receive antibiotics are shown in Supplemental Table 1, available at https://www.AnnFam Med.org/content/19/3/232/ suppl/DC1/. Relative to participants in the control group, participants in the futility and adverse effect groups were less likely to continue to expect antibiotics (unadjusted odds ratio $[\mathrm{OR}]=0.45 ; 95 \% \mathrm{CI}, 0.27$ to $0.75 ; P<.01$, and unadjusted $\mathrm{OR}=0.53 ; 95 \% \mathrm{CI}, 0.32$ to 0.86 ; $P=.01$, respectively).

We refined the multivariate ordinal regression model until it included variables that did not display multicollinearity and until the assumption of proportional odds was met. The final model was a good fit to the data $(P=.38)$ and the model predicted participants' expectations to receive antibiotics better than the intercept-only model $(P<.001)$. Participants 


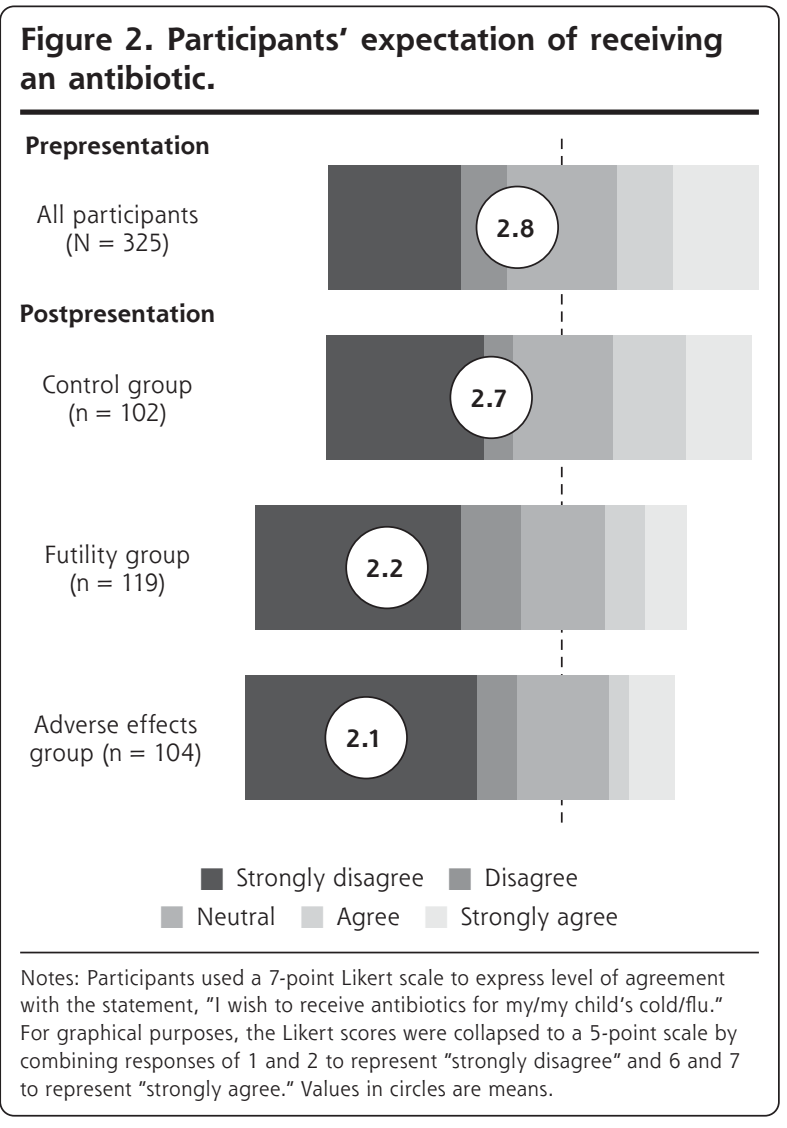

in the futility group (adjusted OR $[\mathrm{aOR}]=0.58 ; 95 \%$ CI, 0.35 to $0.95 ; P=.03)$ and in the adverse effects group $(\mathrm{aOR}=0.44 ; 95 \% \mathrm{CI}, 0.26$ to $0.74 ; \mathrm{P}<.01)$ were less likely to continue to expect antibiotics compared with peers in the control group (Table 2). Males $(\mathrm{aOR}=1.75 ; 95 \% \mathrm{CI}, 1.12$ to $2.74 ; P=.02)$ and participants who reported having a sore throat $(\mathrm{aOR}=1.62$; $95 \% \mathrm{CI}, 1.06$ to 2.48 ; $P=.03$ ) were more likely to continue to expect antibiotics.

\section{Prescribing, Dispensing, and Participant Satisfaction}

Of the 306 participants who completed the postconsultation questionnaire, 30\% (92) reported receiving an antibiotic prescription. There was no significant difference in this proportion between the futility group (31\%), the adverse effects group (28\%), and the control group $(32 \%)\left(\chi^{2}=0.361 ; P=.84\right)$.

Pharmacy dispensing information was available for 304 of the 306 participants who gave consent for their records to be reviewed. Overall, 28\% (84) of these participants were dispensed an antibiotic in the 7 days after their consultation. The proportion did not differ between the futility group (26\%), the adverse effects group (24\%), and the control group (33\%) $\left(\chi^{2}=1.68\right.$, $P=.43$ ). Interestingly, $40 \%$ of participants (37 out of
92) who reported receiving an antibiotic prescription did not have an antibiotic dispensed in the following 7 days, and $32 \%$ of participants ( 27 out of 84 ) who were dispensed an antibiotic did not report receiving an antibiotic prescription after their consultation.

Participants' level of satisfaction with their consultation did not differ across the 3 groups viewing different presentations. The mean (SD) score for satisfaction out of a possible 5 points was $4.7(0.6)$ in the futility group, $4.8(0.5)$ in the adverse effects group, and 4.7 $(0.5)$ in the control group $\left(F_{(2,303)}=0.59, P=.56\right)$.

\section{Factors Associated With Prescribing or Dispensing}

We used binomial logistic regression analyses to determine the effect of various factors on receipt of an antibiotic prescription and on the dispensing of an antibiotic in the week after the consultation. Linearity of the continuous variables (age, perceived severity) was confirmed for each model.

Participants who strongly agreed with the statement "I wish to receive antibiotics for my/my child's cold/flu" after viewing the presentation were significantly more likely to receive an antibiotic prescription $(\mathrm{aOR}=2.69 ; 95 \% \mathrm{CI}, 1.27$ to $5.69 ; P=.01)$ and to have an antibiotic dispensed in the 7 days after the consultation $(\mathrm{aOR}=6.76 ; 95 \% \mathrm{CI}, 2.92$ to $15.67 ; \mathrm{P}<.01)$ (Table 3). (Univariate ORs are given in Supplemental Table 2, available at https://www.AnnFamMed.org/ content/19/3/232/suppl/DC1/.) In addition, participants who reported that earache was a predominant symptom for them or their child were more likely to receive a prescription $(\mathrm{aOR}=2.36 ; 95 \% \mathrm{CI}, 1.30$ to $4.30 ; \mathrm{P}$ $<.01)$, and participants who perceived their/their child's illness as being severe were more likely to have an antibiotic dispensed $(\mathrm{aOR}=1.49 ; 95 \% \mathrm{CI}, 1.12$ to 2.00 ; $P=.01$ for each point increase on the 7-point scale).

\section{DISCUSSION}

Our study shows that providing brief information to patients about either the futility of antibiotic treatment or the potential adverse effects of antibiotics, when they present to their family practitioner with an URTI, halves the patients' expectations for antibiotics. Although the study was not primarily designed to examine antibiotic prescribing, there was no corresponding decrease in this outcome despite the large reduction in participants' expectations for antibiotics. Participants who had strongly expected an antibiotic prescription were more likely to be prescribed an antibiotic, and to have an antibiotic dispensed in the week after their consultation. The receipt of antibioticrelated information before the consultation did not 
detrimentally affect patients' experience of their family practitioner visit. Taken together, our findings show that information about antibiotics was well received and understood by patients at a time when they were unwell and seeking care, but that providing them with this information did not influence their physicians' prescribing behavior.

\section{Strengths and Limitations}

Our findings are novel in providing causal evidence that patients' beliefs and expectations about antibiotics can be modified by provision of information in the family practice waiting room using a brief, low-cost intervention. Second, recruitment of a large sample was conducted at 2 family practices staffed by a total of 18 family practitioners. There was high retention of participants with full data obtained for $90 \%$ of the cohort. Participants were blinded to the aims of the study, and all study personnel were blinded to group allocation. Finally, to increase the validity of outcome data, we used 2 methods to measure family practitioners' prescribing behavior.

The study also had several limitations. Participants were recruited at only 2 family practices, both situated in wealthy, urban areas, serving populations who almost universally have good English proficiency. The study practices had low rates of antibiotic prescribing (30\% overall) compared with other practices in New Zealand. ${ }^{16}$ This difference may limit the generalizability of the intervention effects to other family practices in this country and elsewhere. Our intervention will need implementation testing in a larger number of practices over a prolonged period.

\section{Comparison With Existing Literature}

Our results are in agreement with those of other studies that highlight the impact of patients' beliefs and expectations on inappropriate antibiotic prescribing. ${ }^{13}$ The reduction in patients' expectations that an antibiotic would be prescribed, seen in the intervention groups, can be explained by the Necessity-Concern Framework, which posits that patients' beliefs and behaviors relating to medication can be determined by the balance between the perceived necessity of a medication for maintaining health, and concern about the consequences of taking that medication. ${ }^{14}$ Interestingly, provision of information about the futility of antibiotic
Table 2. Adjusted Odds Ratios of the Likelihood of Continuing to Expect Antibiotics

\begin{tabular}{|c|c|c|c|}
\hline Characteristic & $\begin{array}{l}\text { Proportion } \\
\text { Expecting } \\
\text { Antibiotics, \%a }\end{array}$ & aOR $(95 \% \mathrm{Cl})$ & $P$ Value \\
\hline \multicolumn{4}{|l|}{ Group } \\
\hline Control & 39 & Ref & $\ldots$ \\
\hline Futility & 40 & $0.58(0.35-0.95)$ & .03 \\
\hline Adverse effects & 39 & $0.44(0.26-0.74)$ & $<.01$ \\
\hline \multicolumn{4}{|l|}{ Sex } \\
\hline Female & 36 & Ref & $\ldots$ \\
\hline Male & 48 & $1.75(1.12-2.74)$ & .02 \\
\hline \multicolumn{4}{|l|}{ Age-group } \\
\hline Adult & 40 & Ref & $\ldots$ \\
\hline Child $^{\mathrm{b}}$ & 39 & $0.72(0.44-1.18)$ & .19 \\
\hline \multicolumn{4}{|c|}{ Predominant symptoms } \\
\hline \multicolumn{4}{|c|}{ Sore throat } \\
\hline No & 39 & Ref & $\ldots$ \\
\hline Yes & 39 & $1.62(1.06-2.48)$ & .03 \\
\hline \multicolumn{4}{|l|}{ Cough } \\
\hline No & 33 & Ref & $\cdots$ \\
\hline Yes & 42 & $1.30(0.81-2.11)$ & .28 \\
\hline \multicolumn{4}{|l|}{ Earache } \\
\hline No & 38 & Ref & $\ldots$ \\
\hline Yes & 44 & $1.28(0.79-2.09)$ & .32 \\
\hline Symptom severityc & $\ldots$ & $1.16(0.91-1.47)$ & .22 \\
\hline Degree of concernc & $\ldots$ & $1.15(0.96-1.37)$ & .13 \\
\hline
\end{tabular}

$\mathrm{aOR}=$ adjusted odds ratio; Ref $=$ reference.

Note: Assessed after participants viewed 1 of the presentations, with the postpresentation questionnaire.

Unadjusted.

b Parents provided response for children aged 0 to 7 years presenting with symptoms of upper respiratory tract infection.

Measured on a 7-point scale where higher score indicates greater severity or concern. 
Regardless, we found that participants who strongly expected antibiotics were much more likely to receive an antibiotic prescription.

In general, prescribers overestimate patients' expectations for antibiotics. ${ }^{8}$ This overestimation seems to be driven by the misconception that providing an antibiotic prescription is associated with higher patient satisfaction. ${ }^{19}$ We found no differences between patients who were given an antibiotic prescription and those who were not in their reported level of satisfaction with their consultation.

Clearly, both patients' expectations and prescribers' perceptions of these expectations influence the decision about whether to prescribe an antibiotic. Future efforts to improve antibiotic prescribing need to involve both patients and their clinicians.

\section{Implications for Research and Practice}

This randomized controlled trial shows that patients' expectations for antibiotics can be modified in the family practice waiting room. The results also highlight that the success of any antimicrobial stewardship intervention in reducing prescribing is contingent on prescriber involvement, and they provide support for implementation of this patient education intervention in combination with a complementary prescriberfocused intervention.

To read or post commentaries in response to this article, go to https://www.AnnFamMed.org/content/19/3/232/tab-e-letters.

Key words: patient expectations; respiratory tract infections; antibiotic prescriptions; antimicrobial stewardship education; physician-patient communication; family practice; primary health care; practice-based research

Table 3. Adjusted Odd Ratios of Receiving an Antibiotic Prescription and Having an Antibiotic Dispensed

\begin{tabular}{|c|c|c|c|c|c|c|}
\hline \multirow[b]{2}{*}{ Characteristic } & \multicolumn{3}{|c|}{ Antibiotic Prescription Received } & \multicolumn{3}{|c|}{ Antibiotic Dispensed } \\
\hline & $\begin{array}{c}\text { Unadjusted } \\
\text { Proportion, \% }\end{array}$ & aOR $(95 \% \mathrm{Cl})$ & $P$ Value & $\begin{array}{l}\text { Unadjusted } \\
\text { Proportion, \% }\end{array}$ & aOR $(95 \% \mathrm{Cl})$ & $P$ Value \\
\hline \multicolumn{7}{|l|}{ Group } \\
\hline Control & 31 & Ref & $\ldots$ & 32 & Ref & $\ldots$ \\
\hline Futility & 30 & $0.86(0.46-1.61)$ & .60 & 27 & $1.09(0.56-2.09)$ & .80 \\
\hline Adverse effects & 31 & $0.83(0.43-1.59)$ & .60 & 24 & $0.73(0.36-1.47)$ & .40 \\
\hline Age in years & $\ldots$ & $1.02(1.00-1.04)$ & .10 & $\ldots$ & $1.01(0.99-1.03)$ & .40 \\
\hline Symptom severity & $\ldots$ & $1.26(0.95-1.66)$ & .10 & $\ldots$ & $1.49(1.12-2.00)$ & .01 \\
\hline \multicolumn{7}{|c|}{ Predominant symptoms } \\
\hline \multicolumn{7}{|c|}{ Cough } \\
\hline No & 28 & Ref & $\ldots$ & 29 & Ref & $\ldots$ \\
\hline Yes & 31 & $0.94(0.50-1.76)$ & .80 & 27 & $0.72(0.38-1.38)$ & .30 \\
\hline \multicolumn{7}{|l|}{ Sore throat } \\
\hline No & 31 & Ref & $\ldots$ & 28 & Ref & $\ldots$ \\
\hline Yes & 30 & $0.59(0.34-1.04)$ & .07 & 27 & $0.57(0.31-1.04)$ & .07 \\
\hline \multicolumn{7}{|l|}{ Nasal } \\
\hline No & 29 & Ref & $\ldots$ & 24 & Ref & $\ldots$ \\
\hline Yes & 32 & $0.96(0.54-1.71)$ & .90 & 31 & $1.20(0.66-2.20)$ & .60 \\
\hline \multicolumn{7}{|l|}{ Earache } \\
\hline No & 27 & Ref & $\ldots$ & 25 & Ref & $\ldots$ \\
\hline Yes & 42 & $2.36(1.30-4.30)$ & $<.01$ & 36 & $1.80(0.96-3.41)$ & .07 \\
\hline \multicolumn{7}{|l|}{ Age-group } \\
\hline Adult & 30 & Ref & $\ldots$ & 28 & Ref & $\ldots$ \\
\hline Child & 31 & $1.25(0.68-2.29)$ & .50 & 26 & $1.16(0.62-2.19)$ & .70 \\
\hline \multicolumn{7}{|l|}{ Expect antibiotics ${ }^{a}$} \\
\hline Neutral & 34 & Ref & $\ldots$ & 27 & Ref & $\ldots$ \\
\hline Strongly disagree & 17 & $0.71(0.35-1.45)$ & .30 & 11 & $2.01(0.91-4.44)$ & .08 \\
\hline Disagree & 25 & $0.90(0.34-2.37)$ & .80 & 25 & $1.61(0.56-4.64)$ & .40 \\
\hline Agree & 30 & $1.08(0.46-2.54)$ & .90 & 22 & $2.05(0.78-5.35)$ & .10 \\
\hline Strongly agree & 56 & $2.69(1.27-5.69)$ & .01 & 58 & $6.76(2.92-15.67)$ & $<.01$ \\
\hline
\end{tabular}


Submitted May 6, 2020; submitted, revised, October 4, 2020; accepted October 7, 2020.

Funding support: New Zealand Lottery Health Research Project Grant (Ref. 3715101).

Disclaimer: The views expressed are solely those of the authors and do not necessarily represent official views of the authors' affiliated institutions or funder.

Trial registration: Australia New Zealand Clinical Trials Registry - ID: 12617000744358.

Acknowledgments: The authors wish to thank Blake Rainey for creating the images used in the interventions, Dr Tim Akroyd for overseeing the research at Greenwood Medical Centre, and Professor Elizabeth Broadbent for her guidance with regard to data analysis. We are also grateful to Meihana Douglas and Dana Antunovich for their assistance with data collection.

Supplemental materials: Available at https://www.AnnFamMed. org/content/19/3/232/suppl/DC1/.

\section{References}

1. Cassini A, Högberg LD, Plachouras D, et al; Burden of AMR Collaborative Group. Attributable deaths and disability-adjusted life-years caused by infections with antibiotic-resistant bacteria in the EU and the European Economic Area in 2015: a population-level modelling analysis. Lancet Infect Disease. 2019;19(1):56-66.

2. Duffy E, Ritchie S, Metcalfe S, Van Bakel B, Thomas MG. Antibacterials dispensed in the community comprise $85 \%-95 \%$ of total human antibacterial consumption. J Clin Pharm Ther. 2018;43(1): 59-64.

3. Gulliford MC, Dregan A, Moore MV, et al. Continued high rates of antibiotic prescribing to adults with respiratory tract infection: survey of 568 UK general practices. BMJ Open. 2014;4(10):e006245.

4. McCullough AR, Pollack AJ, Plejdrup Hansen M, et al. Antibiotics for acute respiratory infections in general practice: comparison of prescribing rates with guideline recommendations. Med J Aust. 2017;207(2):65-69.

5. Shehab N, Patel PR, Srinivasan A, Budnitz DS. Emergency department visits for antibiotic-associated adverse events. Clin Infect Dis. 2008;47(6):735-743.

6. Linder JA. Editorial commentary: antibiotics for treatment of acute respiratory tract infections: decreasing benefit, increasing risk, and the irrelevance of antimicrobial resistance. Clin Infect Dis. 2008; $47(6): 744-746$.
7. Fletcher-Lartey S, Yee M, Gaarslev C, Khan R. Why do general practitioners prescribe antibiotics for upper respiratory tract infections to meet patient expectations: a mixed methods study. BMJ Open. 2016;6(10):e012244.

8. Cole A. GPs feel pressurised to prescribe unnecessary antibiotics, survey finds. BMJ. 2014;349:95238.

9. Sirota M, Round T, Samaranayaka S, Kostopoulou O. Expectations for antibiotics increase their prescribing: causal evidence about localized impact. Health Psychol. 2017;36(4):402-409.

10. Ritchie SR, Jayanatha KJ, Duffy EJ, Chancellor J, Allport Z, Thomas MG. Previous antibiotic-related adverse drug reactions do not reduce expectations for antibiotic treatment of upper respiratory tract infections. J Glob Antimicrob Resist. 2017;10:256-260.

11. Coenen S, Francis N, Kelly M, et al; GRACE Project Group. Are patient views about antibiotics related to clinician perceptions, management and outcome? A multi-country study in outpatients with acute cough. PLoS One. 2013;8(10):e76691.

12. Broniatowski DA, Klein EY, Reyna VF. Germs are germs, and why not take a risk? Patients' expectations for prescribing antibiotics in an inner-city emergency department. Med Decis Making. 2015; 35(1):60-67.

13. King LM, Fleming-Dutra KE, Hicks LA. Advances in optimizing the prescription of antibiotics in outpatient settings. BMJ. 2018;363: k3047.

14. Horne R, Weinman J. Patients' beliefs about prescribed medicines and their role in adherence to treatment in chronic physical illness. J Psychosom Res. 1999;47(6):555-567.

15. Ritchie SR, Rakhmanova L, Out-O'Reilly E, Reay S, Thomas MG, Sajtos L. The use of a poster to reduce expectations to receive antibiotics for a common cold. Eur J Clin Microbiol Infect Dis. 2019;38(8): 1463-1469.

16. Health Quality and Safety Commission New Zealand. Atlas of Healthcare Variation on community use of antibiotics. New Zealand Government. Published 2019. Accessed Apr 2019. https://www. hqsc.govt.nz/our-programmes/health-quality-evaluation/projects/ atlas-of-healthcare-variation/community-use-of-antibiotics/

17. Mehrotra A, Linder JA. Tipping the balance toward fewer antibiotics. JAMA Intern Med. 2016;176(11):1649-1650.

18. Dempsey PP, Businger AC, Whaley LE, Gagne JJ, Linder JA. Primary care clinicians' perceptions about antibiotic prescribing for acute bronchitis: a qualitative study. BMC Fam Pract. 2014;15(1):194.

19. Mangione-Smith R, Zhou C, Robinson JD, Taylor JA, Elliott MN, Heritage J. Communication practices and antibiotic use for acute respiratory tract infections in children. Ann Fam Med. 2015;13(3):221-227. 